\title{
O estudo da Acústica Urbana através de Processos Digitais: Novas Abordagens para uma Elaboração Metodológica
}

\author{
The study of Urban Acoustics through Digital Processes: New approaches to developing a Methodology \\ Marcio Nisenbaum \\ Universidade Federal do Rio de Janeiro, Brasil \\ marcionisenbaum@ufrj.br \\ - José Ripper Kós \\ Universidade Federal do Rio de Janeiro, Brasil \\ josekos@ufrj.br
}

\begin{abstract}
This paper aims to discuss different possibilities and methodologies within the field of urban acoustics and the use of digital tools. As an attempt to integrate quantitative methods, such as sound mapping and noise control, with qualitative approaches, such as the soundscape studies, new strategies based on performance oriented design will be addressed. The notion of sonic effect as an important tool for integrating different perspectives will hence be discussed as a possible instrument for structuring generative design experiments. A theoretical framework and a possible design workflow are further drawn to possibly articulate future studies.
\end{abstract}

Keywords: Soundscape, Noise Mapping, Sonic Effect, Acoustics, Computational Design

\section{Introdução}

Os estudos acerca das condições acústicas das cidades compreendem desde o mapeamento e controle de ruídos até a análise das paisagens sonoras (soundscapes). Embora a preocupação com a poluição sonora tenha aumentado nos últimos anos, a acústica, no âmbito do urbanismo, ainda é um tema pouco discutido na academia e negligenciado no processo de planejamento das cidades.

A falta de estudos mais aprofundados sobre questões de acústica urbana se evidencia no próprio campo de representações e técnicas digitais. Mesmo com o desenvolvimento de mídias capazes de lidar com o processamento de comportamentos sonoros complexos, percebemos na área de pesquisa do design computacional e seus campos investigativos uma priorização dos aspectos formais/visuais dos objetos arquitetônicos e urbanos, desconsiderando alguns fatores relacionados aos nossos outros sentidos, entre eles, o som.

A presente pesquisa se propõe a refletir sobre os sons urbanos e as possibilidades de representação, simulação e avaliação sonora mediadas pelas técnicas digitais. Entendemos que alguns processos computacionais como "parametrização", "sistemas generativos", "gramática da forma", entre outros, podem abrir novos caminhos no estudo da acústica das cidades.

Apresentaremos nesse artigo algumas questões teóricas envolvendo mapeamento de ruídos e o estudo das paisagens sonoras - a partir da obra de Schafer (1994) - buscando uma articulação entre essas áreas investigativas aparentemente antagônicas. Discutiremos o atual processo de cartografia sonora e colocaremos em debate possibilidades de mapeamento e simulação mais dinâmicas.

$\mathrm{Na}$ tentativa de integrar avaliações quantitativas e qualitativas do som, recorreremos ao conceito de "efeito sonoro" (sonic effect), a partir da obra de Augoyard e Torgue (2005). A partir dessa noção buscaremos entender a ideia de "performance acústica", que pode auxiliar na estruturação de experimentos generativos com ferramentas digitais em projetos urbanos.

\section{O mapeamento de ruídos}

A análise da poluição sonora e o controle de ruídos constituem atualmente o campo de pesquisa mais proeminente dentre os estudos sobre a acústica urbana, na medida em que a questão sonora das cidades se tornou um problema de saúde pública. De acordo com a OMS, a poluição sonora é hoje o tipo de poluição que atinge o maior número de pessoas no planeta depois da poluição do ar e da água (GUEDES, 2008). É comprovado que a exposição à níveis elevados de ruídos podem causar danos ao aparelho auditivo e provocar distúrbios emocionais (NIEMEYER, 2014).

No Brasil, o único instrumento regulatório que trata da questão acústica é a norma NBR 10151- Avaliação do Ruído 
em Áreas Habitadas Visando o Conforto da Comunidade (ABNT/2000) que estabelece níveis máximos de ruídos ambientais em áreas consolidadas, considerando níveis de pressão sonora aceitáveis para o dia e noite.

\begin{tabular}{|c|c|c|}
\hline Tipos de áreas & Diurno & Noturno \\
\hline Áreas de sítios e fazendas & 40 & 35 \\
\hline $\begin{array}{c}\text { Área estritamente residencial } \\
\text { urbana ou de hospitais ou de } \\
\text { escolas }\end{array}$ & 50 & 45 \\
\hline $\begin{array}{c}\text { Área mista, predominantemente } \\
\text { residencial }\end{array}$ & 55 & 50 \\
\hline $\begin{array}{c}\text { Área mista, com vocação } \\
\text { commercial e administrativa }\end{array}$ & 60 & 55 \\
\hline $\begin{array}{c}\text { Área mista, com vocação } \\
\text { recreacional }\end{array}$ & 65 & 55 \\
\hline $\begin{array}{c}\text { Área predominantemente } \\
\text { industrial }\end{array}$ & 70 & 60 \\
\hline
\end{tabular}

Tabela 1: Nível de critério de avaliação NCA para ambientes externos, em dB(A) - decibéis. Fonte: ABNT NBR 10151.

Enquanto no Brasil não há exigência deestudos deimpacto acústico nem mesmo para infraestruturas e rodoviárias de grande porte, na Europa, através da diretiva 2002/49/CE, mapas estratégicos de ruídos passaram a ser obrigatórios em busca de avaliações mais precisas da poluição sonora nas cidades (Niemeyer, Cortês, 2013). Os mapas permitem uma representação espacial precisa, evidenciando espaços mais comprometidos pela poluição do som, indicados por gradientes de cores baseados em informações numéricas. A elaboração dos mapas é exigida para aglomerações com mais de 250.000 habitantes e áreas com atividades potencialmente capazes de gerar poluição sonora, se tratando de um instrumento de grande relevância para o planejamento urbano.

Um ponto interessante da diretiva é a padronização dos métodos de avaliação impostos aos países da União Europeia para determinação dos níveis de ruído. Para isso, institui-se os índices Lden (indicador de ruído dia-fim-de-tarde-noite) para avaliar o incômodo geral, e o Lnight (indicador de ruídos noturnos) para avaliar as perturbações noturnas. Se os valores limites estabelecidos forem ultrapassados, os Estadosmembros devem traçar planos de ação para minimizar a poluição sonora.

No que se refere ao processo de cálculo e análise de ruídos, as tecnologias digitais têm se desenvolvido de forma significativa, tanto através da elaboração de uma diversidade de softwares de mapeamento acústico por empresas privadas, mas também pela aplicação desses recursos em projetos de pesquisas e produções acadêmicas. Dessa forma, processos de mapeamento sonoro tradicionais, feitos in loco através de processos manuais, vêm sendo substituído ou complementado por tais ferramentas.

A vantagem da utilização das ferramentas digitais no processo de mapeamento é, além da precisão da avaliação, a possibilidade de predição de cenários futuros. No Brasil, muitos estudos acadêmicos têm utilizado tais instrumentos, como a pesquisa de Guedes (2008), que estudou a influência da forma urbana no ambiente sonoro do bairro Jardins, em Aracaju, e Lopes (2003), que estudou o mapeamento sonoro na área central de Florianópolis. Nessa mesma linha, o projeto de pesquisa "Conforto Acústico no ambiente construído" realizado pelo PROARQ-UFRJ, sob a orientação da professora Maria Lygia Niemeyer, também tem alcançado resultados relevantes enfocando as áreas das Vargens, no Rio de Janeiro.

Dentre os softwares de mapeamento disponíveis destacam-se programas como o Predictor-Lima da Empresa Softnoise Gmbh, Mithra da Empresa 01dB-Stell, SoundPLAN da Empresa Braunstein + Berndt $\mathrm{GmbH}$, entre outros (Guedes, 2008). Algumas iniciativas como o projeto OrbiGIS desenvolvem plataformas abertas vinculada à tecnologia GIS com a intenção de disponibilizar o processo de mapeamento de ruídos de forma gratuita. O processo de cálculo algorítmico é semelhante nos diferentes softwares, tendo como variáveis as fontes sonoras, as características topográficas, as barreiras acústicas, os processos de reflexão, as superfícies, os materiais refletores e absorventes etc.

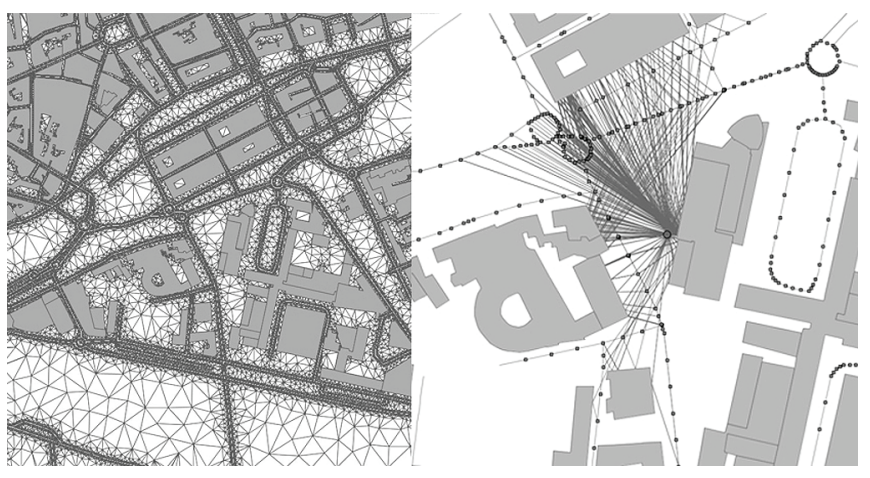

Figura 1: Processo de triangulação da malha e a simulação de reflexão (fonte: http://noisemap.orbisgis.org/) .

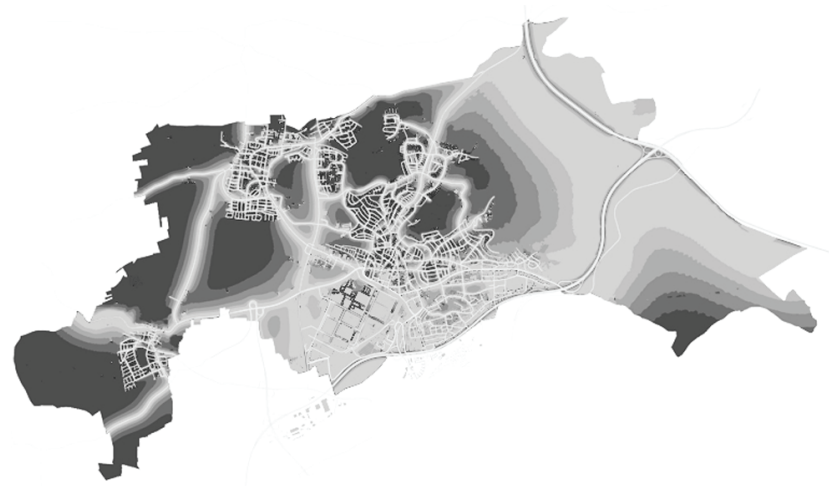

Figura 2: Mapa de ruído final (fonte: http://www.soundplan.eu).

Como um desdobramento do processo digital de mapeamento de ruídos, espera-se que as novas possibilidades 
e ferramentas de design digital possam ainda potencializar esses avanços, introduzindo, por exemplo, o pensamento e processo paramétrico. A utilização dos parâmetros gerados pelos mapas de ruídos como inputs de otimização acústica em função da morfologia urbana e fontes de ruídos parece ser um caminho de pesquisa interessante. A aplicação de métodos evolutivos de simulação (como algoritmos genéticos) podem auxiliar em experimentos nesse sentido.

Entretanto, um questionamento cabível em relação à produção de mapas de ruído seria sua incapacidade de distinção entre sons que seriam agradáveis e sons indesejados. Da mesma forma que num projeto urbano a mitigação de ruídos é almejada em algumas circunstâncias, alguns sons da natureza ou até mesmo sons artificias (que contribuam para identidades locais) podem ser apreciados, e nesse caso seus atributos não são quantificados nos mapas. Nesse sentido, no processo de design sonoro, outros campos investigativos da acústica devem também ser levados em consideração.

\section{Paisagens sonoras: um enfoque qualitativo}

Enquanto o estudo da poluição sonora busca formas de mapear e controlar ruídos, o campo investigativo das paisagens sonoras propõe um outro tipo de abordagem, na qual os sons podem ser interpretados de forma qualitativa. Para Rêgo (2008, p.17): "Os sons e os demais elementos imateriais, como a luz e o calor, juntam-se aos elementos materiais, formas e texturas, para qualificar os ambientes. (...) Isto só é possível porque, entre o silêncio e o ruído, existe uma infinita variedade de sons e de escutas".

Esse enfoque, que busca evidenciar a importância cultural dos sons urbanos e naturais, tem sua gênese na obra de Murray Schafer, intitulada "The soundscape: our sonic environment and the tuning of the world". O termo soundscape (traduzido para paisagem sonora) cunhado pelo autor, se refere à contribuição dos sons para a construção do conceito de paisagem, que vai muito além da questão visual.

Na obra em questão, Schafer cria um sistema de leitura da paisagem através da categorização de tipos sonoros. Assim, conceitos como marco sonoro e ambiente sonoro (uma espécie de relação figura-fundo do som), além de outros recursos de leitura da paisagem, são utilizados na tentativa de diferenciar sons de ruídos, dependendo do contexto. Sua obra é considerada uma grande contribuição para o estudo do som ambiental, tanto pelo caráter historiográfico quanto pela concepção inovadora de um sistema qualitativo de interpretação sonora.

A produção de Schafer, assim como uma série de outras publicações influenciadas pelo autor, lançaram as bases para um campo disciplinar denominado Acústica Ecológica, que busca relacionar o som das cidades com as questões ambientais. A criação do World Forum for Acoustic Ecology (WFAE), associação que promove estudos científicos, sociais e culturais dos aspectos sonoros em diversos ambientes mundiais contribuiu para a proliferação do estudo das paisagens sonoras (Rego, 2006). Alguns instrumentos de natureza qualitativa nasceram desses estudos como os Soundwalks (Figura 3). Trata-se de passeios por determinadas áreas onde pesquisadores se concentram na interpretação das diferentes fontes sonoras e buscam representá-las através de gravações e também de forma gráfica, através de mapas de eventos sonoros.

Exemplos interessantes de mapas de paisagens sonoras são também os de natureza colaborativa, onde cidadãos gravam sons que consideram importantes e disponibilizam através de plataformas online georreferenciadas. Tais manifestações são mais recentes e obviamente influenciadas pelas novas possibilidades tecnológicas. Através de mapas colaborativos, determinados sons das cidades podem ser evidenciados, significando que possuem valor cultural para determinada comunidade.

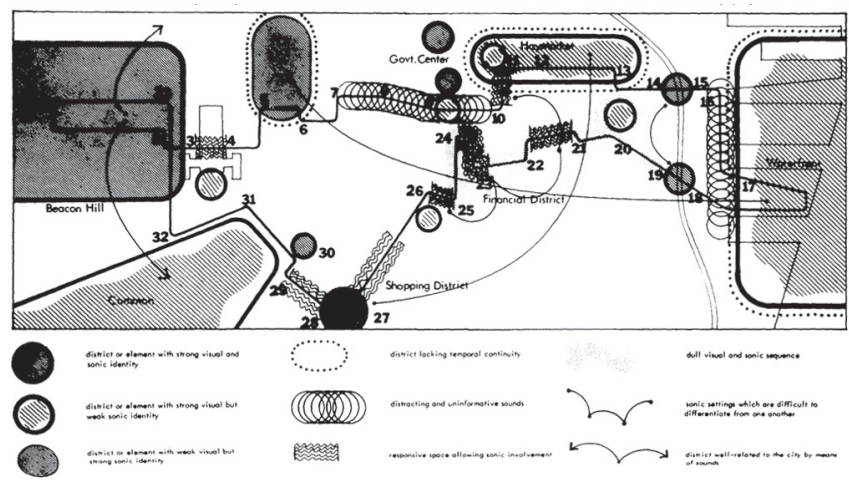

Figura 3: exemplo de Soundwalk realizado por Michael Southworth em Boston. Fonte: Schafer (1994)

\section{A articulação de enfoques: a noção de performance acústica}

Enquanto o mapeamento de ruídos estabelece uma leitura a partir de índices sonoros, o estudo das paisagens sonoras envolve questões de natureza fenomenológica e ambiental. A articulação dessas duas abordagens a princípio parece ser muito difícil, mas também instiga novos caminhos investigativos, podendo se estender a debates no campo do design computacional. A possibilidade de estruturar parâmetros qualitativos e métodos de aferição em modelos de simulação ainda parece ser um grande desafio, especialmente na escala do projeto urbano.

Nesse contexto, pode ser importante refletir sobre o conceito de performance e entender melhor o processo de "projeto orientado pela performance". Para Oxman (2008), no processo de design computacional baseado na ideia de performance "...a ênfase se desloca de fazer uma forma para encontrar uma forma (form-finding) baseada em técnicas generativas". Nesse quadro, um novo tipo de designer é idealizado, que passa de um indivíduo controlador de formas para um agente controlador de processos ou regras. $O$ foco do projeto passa a não ser mais o objeto final, e sim, o conjunto de 
variáveis envolvidos na concepção projetual.

Ainda segundo Oxman (2008), no processo de design orientado pela performance, três componentes devem ser integrados no processo de design: um modelo generativo que pode ser transformado em função de inputs; um processo de avaliação que pode ser integrado ao modelo e gerar as transformações; e um sistema que promova interatividade, sendo o designer um moderador paramétrico/algorítmico dos vários processos envolvidos.

No campo da acústica, a utilização de um modelo de trabalho orientado pela performance (poderíamos usar o termo sound-oriented-design) pode ajudar no entendimento das interações dos sons com a morfologia urbana. Porém, uma aplicação pragmática do conceito pode reduzir as avaliações acústicas de um projeto unicamente a critérios de desempenho ou eficiência. Um edifício, por exemplo, poderia "performar" melhor que outro ao reduzir os ruídos provenientes do exterior, ou minimizar os sons entre os ambientes internos. Uma determinada quadra em uma cidade específica, da mesma forma, pode ter sua performance mensurada se a morfologia urbana contribuir para que os ruídos sejam atenuados em determinadas áreas de uso residencial. Nessas situações, a performance pode ser medida por índices, parâmetros e projeções, facilitando um processo de parametrização.

Já uma abordagem da acústica que incorpore noções qualitativas exige uma leitura de performance por outro viés. Seria difícil, por exemplo, mensurar de forma objetiva a performance de um som ambiental cuja apreciação varie em diferentes culturas. Nesse sentido, devemos nos apropriar do conceito de performance com uma certa cautela e refletir mais sobre suas possibilidades semânticas.

Para Duarte e Noronha (2013), uma interpretação mais abrangente deveria tratar o conceito de performance como um processo, uma ação, um desempenho através de um corpo, contexto e tempo. Essa noção corporal pode ser relativizada em diferentes níveis, como o "corpo" sendo presença física, o "corpo" como o próprio objeto edificado assim como o "corpo" do designer do projeto. Essa visão se aproxima da abordagem de Leatherbarrow (2009), que em seu livro "Architecture Oriented Otherwise" dedica um capítulo inteiro à procura de novas interpretações para o conceito de performance. Para o autor (apud Hensel, 2013): "quando um edifício é entendido como o locus da performance (e não de soluções funcionais), ele pode ser visto como preparação e resposta; um conjunto de condições que não só antecipam ocorrências mas reagem a elas, através da antecipação no primeiro caso e participação no segundo."

Novas abordagens acerca do conceito de performance podem abrir caminhos para a construção de uma noção mais ampla do que seria performance acústica. Entender a morfologia urbana como um corpo que age e reage a estímulos sonoros pode facilitar o desenvolvimento de uma metodologia de design sonoro mais dinâmica, e auxiliar no desenvolvimento de sistemas generativos. Nesse sentido, para não reduzir a noção da performance exclusivamente à questão de otimização acústica, métodos de qualificar o som devem ser investigados e devem fazer parte do processo de design.

\section{Metodologia Híbrida: o efeito sonoro}

Como vimos anteriormente, encontramos na obra de Schafer as primeiras tentativas de qualificação e classificação do som, na tentativa de estabelecer grupos e tipos sonoros. Através de conceitos como fundo sonoro, evento sonoro, sinal sonoro e marco sonoro, o autor buscou estabelecer uma hierarquia do som ambiental. Schafer também foi o responsável pela introdução da ideia de um pensamento de design sonoro, ao defender que profissionais que lidem com as paisagens sonoras deveriam exercitar sua capacidade de ouvir, assim como adotar e desenvolver metodologias de trabalho como os soundwalks.

Outra obra fundamental no campo da acústica ecológica é o livro "Sonic Experience: a Guide for everyday sounds" de Jean-François Augoyard e Henry Torgue, resultado da pesquisa do grupo Cresson (Centre for Research on Sonic Space and the Urban Environment). A pesquisa desses autores vai um pouco além da abordagem de Schafer ao introduzir o conceito de "efeito sonoro" (sonic effect). A interpretação dos sons urbanos como efeitos sonoros parece se aproximar da noção de performance, uma vez que o som não é considerado um objeto independente, mas inserido em um milieu, um contexto, influenciando e sendo influenciado, agindo e reagindo.

Essa abordagem é interessante pois além de lidar com questões de percepção subjetiva do som, tema que já vinha sendo discutido por Schafer, a forma da cidade como componente transformador da paisagem sonora volta a ganhar importância. Para Augoyard e Torgue (2005, p.10), a maioria dos efeitos sonoros depende do seu contexto espacial: “(..) sem uma organização particular e uma morfologia, não haveria reverberação (reverberation), ressonância (ressonance), efeito de corte (cut out), onipresença (ubiquity) ou filtragem (filtration). A acústica aplicada demonstra como espaço, volume, forma e materiais determinam a propagação dos sons."

Ainda segundo os autores, a noção de efeitos sonoros funciona como uma ferramenta que integra processos de percepção e ação, observação e concepção, análise e criação (Augoyard \& Torgue, 2005). Nesse sentido, o enfoque adotado pelos autores busca reduzir a distância entre campos disciplinares atualmente dissociados, integrando avaliações qualitativas e quantitativas, as ciências sociais, os estudos urbanos etc. $\mathrm{O}$ efeito sonoro, nesse contexto, nasceria da interseção do som ambiental, da representação do som de uma comunidade específica, além da percepção individual.

Os autores estruturam a obra através de 66 efeitos sonoros, dando especial ênfase a 16 (na figura 4, aparecem em negrito), denominados efeitos maiores (major effects). Cada efeito é analisado sob diferentes óticas, levando em conta interpretações em campos como psicologia, arquitetura, música etc. 
Através dos estudos dos efeitos sonoros, a noção de performance acústica ganha novos significados. "Performar" nesse contexto não significa simplesmente reduzir a questão sonora à eficiência acústica, mas também poder trabalhar com nuances, gradientes, efeitos de reverberação, filtragem etc. A construção de um léxico sonoro definida pelos autores auxilia no processo de design orientado pelo som e cria elementos qualitativos de avaliação, uma espécie de gramática da forma sonora em função da morfologia urbana. Esses elementos podem se transformar em parâmetros em uma simulação generativa, atuando como inputs cuja interação com outros componentes pode gerar resultados interessantes, num processo baseado em regras definidas pelo "designer sonoro".

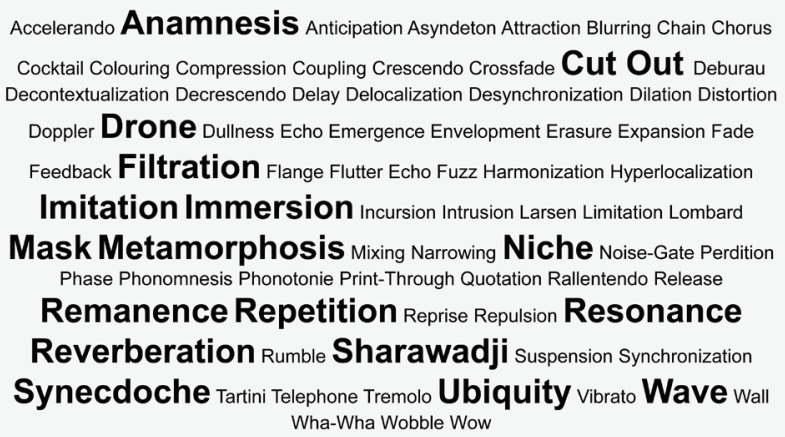

Figura 4: Nuvem de palavras com os efeitos sonoros de Augoyard e Torgue. Fonte (editada): Augoyard e Torgue, 2005.
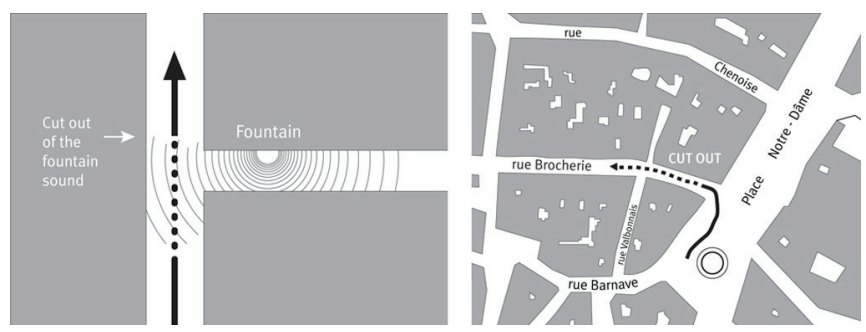

Figura 5: Exemplo morfológico do efeito cutout. Fonte: Augoyard e Torgue, 2005.
Um exemplo de experimentação digital que se baseou no estudo de efeitos sonoros de Augoyard e Torgue é a instalação desenvolvida pelo arquiteto Brady Peters, exibida na conferência SmartGeometry, em 2008. Utilizando softwares paramétricose fabricaçãodigital, Peters buscou alcançar diferentes nuances sonoras no espaço, através de variações de intensidades acústicas geradas pela forma da instalação e pelos materiais utilizados. Assim, conseguiu caracterizar espaços através de atributos sonoros, como "monótonos", "amplificados", "de transição" etc.

O trabalho de Peters pode ser considerado pioneiro não só por explorar um processo de design orientado pela performance acústica, mas por conseguir utilizar efeitos sonoros como instrumentos de design, através de processos de avaliação e simulação digitais. A transposição da lógica do seu trabalho para a escala do projeto urbano parece ser um grande desafio, posto que o número de variáveis é muito maior, mas poderia significar uma contribuição relevante para o campo dos estudos da acústica urbana, e inspirar novas elaborações metodológicas.

Nesse sentido, na tentativa de estruturar um modelo de trabalho que contemple as questões acústicas, podemos recorrer a Lawson (2005), que propôs um sistema metodológico baseado na tríade "análise, síntese e avaliação". No processo proposto pelo autor, um projeto nasce a partir de análises, de identificação de problemas, de obtenção e gerenciamento de informações, de definições conceituais, critérios, etc. A segunda fase projetual, de "síntese", seria o momento de criação, seja através de processos mais intuitivos ou sistemáticos, ou a combinação dos dois métodos. Já a última etapa, de "avaliação", seria a etapa de reflexão, teste de soluções, aferição das metas e critérios idealizados. Cabe ressaltar que as relações entre essas as etapas nem sempre são sequenciais, apresentando muitas vezes características dinâmicas e flexíveis, com limites tênues e momentos de feedbacks e loops (Andrade, Ruschel \& Moreira, 2011).

No âmbito do design acústico das cidades, esse modelo metodológico pode ser tomado como base para o esboço de um framework que inclua os atributos sonoros num processo de projeto urbano, em diferentes escalas. A intenção do framework apresentado (Figura 6) é evidenciar, nas diferentes etapas de projeto, tanto as novas possibilidades instrumentais no campo das arquiteturas digitais, quanto as diferentes formas de interpretação e manipulação de elementos sonoros, podendo servir como base para o desenvolvimento de um sistema paramétrico.

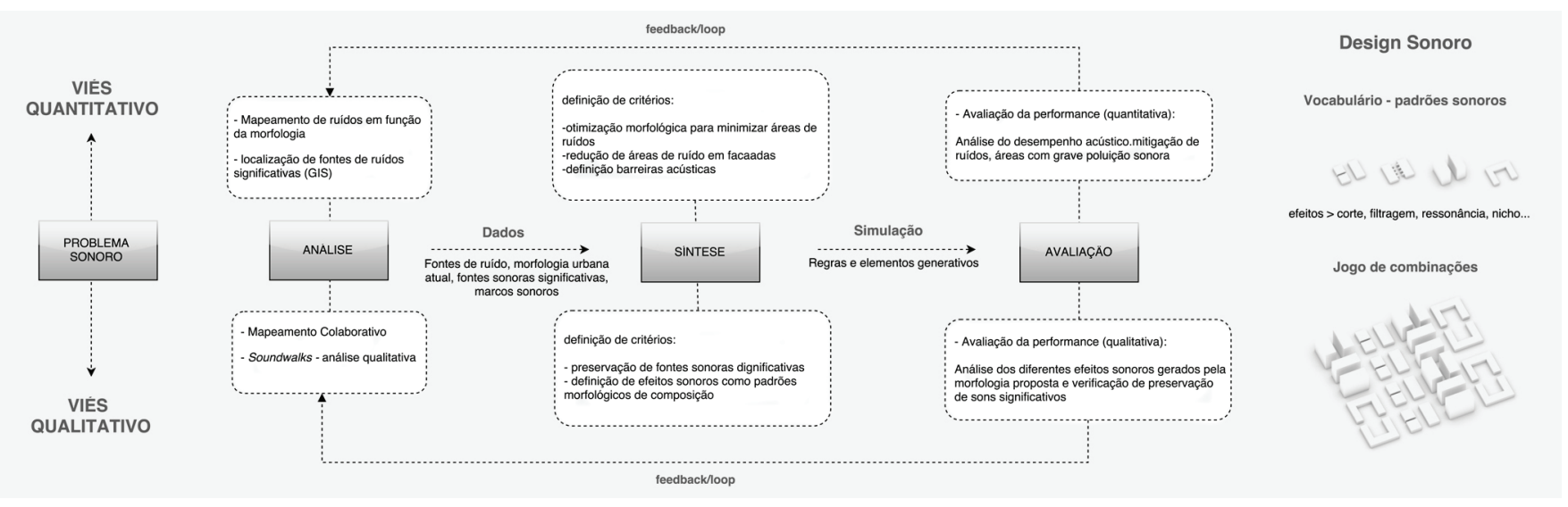

Figura 6: framework idealizado para um processo de design orientado pela performance acústica. 
No modelo proposto, os processos analíticos devem contemplar tanto o mapeamento de ruídos desenvolvido através de softwares, quanto o mapeamento qualitativo, através de soundwalks e mapas colaborativos. Baseado nos dados coletados, a fase de síntese projetual pode conjugar o processo de otimização acústica (através de redução das áreas de ruídos em fachadas e áreas de interesse especial) com as intenções compositivas sonoras. Nesse sentido, o processo de parametrização pode ser estruturado a partir de regras generativas derivadas do vocabulário introduzido por Augoyard e Torgue. Os efeitos sonoros podem ser transformados em padrões espaciais sonoros, articulados num sistema de gramática da forma (ou gramática dos sons) que permita um "jogo" com diversas combinações. Tais combinações permitirão a geração de morfologias diversas, que podem ser analisadas através de índices de performance, tanto no sentido de se chegar ao melhor desempenho acústico, quanto no desenvolvimento de uma morfologia urbana que produza variações sonoras interessantes.

\section{Conclusões e desdobramentos da pesquisa}

As diferentes abordagens em torno dos estudos sonoros revelam que a questão acústica das cidades vai muito além do controle de ruídos. Embora tenha havido considerável evolução tecnológica no processo digital de mapeamento da poluição sonora, cabe aos profissionais envolvidos com questões acústicas buscarem novas metodologias de projeto, considerando também os aspectos qualitativos do som.

A partir das reflexões teóricas e metodológicas apresentadas nesse artigo, entendemos que o conceito de "efeito sonoro", baseado na obra de Augoyard e Torgue, demonstra uma evolução significativa na construção de uma compreensão mais abrangente do som ambiental. Considerando os pressupostos introduzidos por Oxman acerca do "design orientado pela performance", a noção de "efeito sonoro" pode auxiliar na criação de instrumentos para o desenvolvimento de modelo generativos, de critérios avaliativos e de possibilidades de interação do designer com o projeto acústico. Nesse sentido, nasce a possibilidade de um processo de projeto mais dinâmico, articulando controle de ruído, morfologia urbana e manipulação qualitativa dos sons ambientais.

A intenção do framework apresentado, longe de ser um modelo de aplicação fechado, é investigar possibilidades e abrir caminhos para estudos mais aprofundados das diferentes etapas de projeto que lidem com questões acústicas. Uma próxima fase da pesquisa seria justamente se debruçar sobre um desses aspectos e estruturar um protótipo digital - uma ferramenta que auxilie projetos urbanos ao incluir a preocupação com os sons ambientais.

\section{Agradecimentos}

Agradecemos ao Comitê Internacional SIGRADI pelas sugestões que contribuíram para a estruturação da base para este modelo, ao
Programa de Pós-Graduação em Urbanismo da Universidade Federal do Rio de Janeiro (UFRJ), e à CAPES pela viabilidade financeira.

\section{Referências}

Andrade, M., Ruschel, R. \& Moreira, Daniel. (2011). O processo e os métodos. In: O processo de projeto em arquitetura: da teoria à tecnologia. São Paulo: Oficina de textos.

Augoyard, J.F. \& Torgue, H. (2005). Sonic Effects: A Guide To Everyday Sounds. Montreal: McGill-Queen's University Press.

Cortês, Marina Medeiros \& Niemeyer, Maria Lygia Alves de (2013). O potencial da utilização da ferramenta de mapa de ruído em diferentes escalas de análise. In: XII Encontro Nacional de Conforto no Ambiente Construído e VIII Encontro Latinoamericano de Conforto no Ambiente Construído. Brasília: Universidade de Brasilia. 1,155-164. Duarte, V. G. \& Noronha, M. P. (2011) Performance e arquitetura: uma transmutação conceitual a partir do estudo do edifício da fundação iberê camargo, de Álvaro siza. In: II Congresso Internacional do Curso de hsitória da UFG/Jataí, 2011, Jataí.

Guedes, Italo César Montalvão. (2005). Influência da forma urbana em ambiente sonoro: um estudo no bairro Jardins em Aracaju Campinas: Unicamp.

Hensel, Michael. (2013). Performance oriented Architecture. Rethinking Architecture and the Built Environment. Londres: Wiley. Lawson,B. (2005). How designers think: the design process demystified. (4th ed.) Oxford: Elsevier.

Leatherbarrow, David. (2009) Architecture oriented otherwise. Nova Iorque: Princetown Architectural Press.

Nardi, Aline Souza Lopes Ventura. (2003). Mapeamento Sonoro em Ambiento Urbano. Estudo de caso: área central de Florianópolis. Florianópolis: UFSC.

Oxman, Rivka. (2008). Performance-based Design: Current Practices and Research Issues. International Journal of Architectural Computing, 1 (06), 1-17.

Peters, Brady. (2010) Acoustic Performance as a Design Driver: Sound Simulation and Parametric Modeling using SmartGeometry. Brady Peters. International Journal of Architectural Computing, 3 (8), 337358.

Rego, A. Q. ; Niemeyer \& Maria Lygia Alves de. (2014) O uso da cartografia sonora na avaliação da transformação das paisagens sonoras devido à implementação do plano estratégico urbano das Vargens, Rio de Janeiro, brasil. In: iii enanparq: Arquitetura, cidade e projeto: uma construção coletiva. Campinas: Mackenzie/ PUCCampinas, 1, 1-11.

Rêgo, Andréa Queiroz. (2006). Paisagens sonoras e identidades urbanas: os sons nas crônicas cariocas e as transformações do bairro de Copacabana (1905-1968) Rio de Janeiro: UFRJ, 2006. (Tese de Doutorado)

Schafer, R. Murray. (1994). The soundscape - our sonic environmental and tuning of the world (2nd.ed). Rochester: Destiny Books. 